\title{
Homogeneous Modification of Sugarcane Bagasse by Graft Copolymerization in Ionic Liquid for Oil Absorption Application
}

\author{
Ming-Jie Chen, ${ }^{1,2}$ Xue-Qin Zhang, ${ }^{2}$ Chuan-Fu Liu, ${ }^{2}$ and Qing-Shan Shi ${ }^{1}$ \\ ${ }^{1}$ State Key Laboratory of Applied Microbiology Southern China, Guangdong Provincial Key Laboratory of \\ Microbial Culture Collection and Application, Guangdong Institute of Microbiology, Guangzhou 510070, China \\ ${ }^{2}$ State Key Laboratory of Pulp and Paper Engineering, South China University of Technology, Guangzhou 510640, China
}

Correspondence should be addressed to Qing-Shan Shi; shiqingshan@hotmail.com

Received 7 April 2016; Accepted 4 August 2016

Academic Editor: Jun Yang

Copyright (C) 2016 Ming-Jie Chen et al. This is an open access article distributed under the Creative Commons Attribution License, which permits unrestricted use, distribution, and reproduction in any medium, provided the original work is properly cited.

\begin{abstract}
Sugarcane bagasse, lignocellulosic residue from the sugar industry, is an abundant and renewable bioresource on the earth. The application of ionic liquids in sugarcane bagasse biorefinery is gaining increasing interest. The homogeneous modification of sugarcane bagasse by free radical initiated graft copolymerization of acrylate monomers using 1-allyl-3-methylimidazolium chloride as solvent was performed. A variety of sugarcane bagasse graft copolymers with different weight percent gain were prepared via adjusting the monomer dosage. FT-IR studies confirmed the success in attaching the poly(acrylate) side chains onto sugarcane bagasse. Oil absorbency studies suggested that the sugarcane bagasse graft copolymers were potential biobased materials for effective treatment of ester-based oils. SEM studies showed that the sugarcane bagasse graft copolymers displayed a dense morphology structure. Thermogravimetric analysis demonstrated that the thermal stability of sugarcane bagasse decreased after the homogeneous modification by the graft copolymerization. The present study provides an alternative strategy to convert sugarcane bagasse into a value-added functional biobased material.
\end{abstract}

\section{Introduction}

There is growing interest in turning agroindustrial waste biomass into valuable chemicals and materials. The initial interest in this biomass refinery originates from the challenges of resource depletion and waste accumulation [1]. Biorefinery offers the possibility of replacing traditional fossil resources with natural bioresources to manufacture industrial and daily products. Sugarcane bagasse is a typical lignocellulosic waste residue produced by sugar industry. It is estimated that approximately $1.5 \times 10^{8}$ dry tons of sugarcane bagasse is produced worldwide annually [2]. It is commonly used as a primary fuel source for sugar mills and the production of pulp, paper, and boar in substitution of wood [3]. However, there is a potential to make better use of the renewable resource by producing novel functional biobased materials.

Modification by graft copolymerization is an effective approach tailoring the material properties of lignocelluosic biomass. It is achieved by attaching side chains (graft) onto the biomass substrate. Functional surfaces, for example, $\mathrm{pH}$ responsive [4], hydrophobic [5], and antimicrobial [6], of lignocellulosic biomass have been created by the graft approach. However, the cell wall of lignocellulosic biomass is a highly crystalline composite of cellulose, lignin, and hemicellulose, which makes it insolvable in any traditional solvent systems. The modification of the biomass has to be operated at heterogeneous reaction mediums, which achieve low efficiency. To achieve efficient modification of the biomass, the application of a good solvent is mandatory [7].

Ionic liquids, with strong hydrogen bond donor-acceptor ability, are found to be excellent solvents for lignocellulosic biomass $[8,9]$. The application of ionic liquids in biomass refinery is booming over the past decade, due to the designable properties of ionic liquids to meet the challenges of sustainable chemistry [10]. Homogeneous acylation of lignocellulosic materials has been carried out in ionic liquids, which is demonstrated to be an advanced technique with higher degree of substitution ( 94\%) than the heterogeneous acylation [11]. The dissolution of biomass destroys 

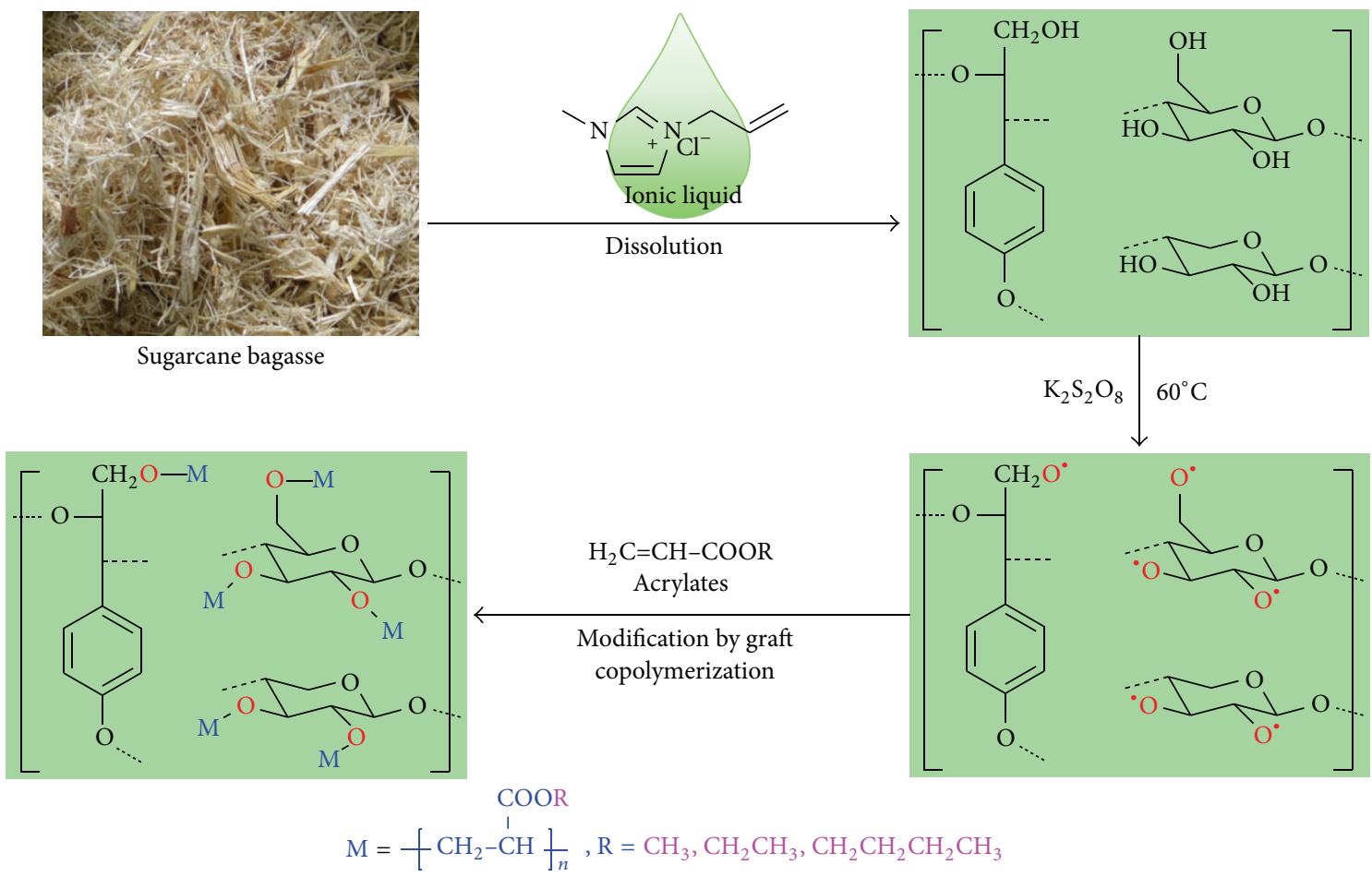

FIGURE 1: Chemical processes for sugarcane bagasse modification by free radical initiated graft copolymerization.

the crystalline structure of the material and releases the hydroxyl groups from hydrogen bonding, thus making the biomass accessible to chemical reagents. Importantly, the components, namely, cellulose, hemicellulose, and lignin, of lignocellulosic biomass have equal opportunities in reaction with anhydrides using 1-butyl-3-methylimidazolium chloride as medium [12]. The development of homogeneous modification of biomass using ionic liquids as mediums has set up an advanced strategy for preparation of novel biobased functional materials.

The homogeneous modification of wood by free radical initiated graft copolymerization of acrylamide using 1-(2hydroxyethyl)-3-methylimidazolium chloride as medium is performed in a previous study [13]. It is demonstrated that the ionic liquid is a more effective reaction medium for the graft copolymerization than water [13]. However, 1-(2-hydroxyethyl)-3-methylimidazolium chloride is hydroxyl group containing ionic liquid, which is not a suitable solvent for the free radical initiated graft copolymerization of sugarcane bagasse, since the hydroxyl group is the reactive site (Figure 1) in the graft copolymerization [14]. On the other hand, the previous research is limited in the graft copolymerization of acrylamide [13], which is a hydrophilic monomer, while the introduction of hydrophobic side chains to the lignocellulosic biomass is mandatory in most situations.

In the present study, the graft copolymerization of hydrophobic acrylate monomers onto sugarcane bagasse was achieved using 1-allyl-3-methylimidazolium chloride (AMIMCl) as reaction medium. The chemical structure, morphology, thermal stability, and oil absorption capacity of the sugarcane bagasse graft copolymers were studied. It was suggested that homogeneous graft copolymerization using ionic liquid as solvent was an advanced technique to transform sugarcane bagasse into value-added biobased materials.

\section{Material and Methods}

2.1. Materials. Sugarcane bagasse was obtained from Guangxi Guitang (Group) Co. Ltd. (Guigang, China). It was washed with hot water $\left(90^{\circ} \mathrm{C}\right)$ for three times and ground to prepare 40-60-mesh size particles. The ground sugarcane bagasse was dewaxed by toluene-ethanol $(2: 1 \mathrm{v} / \mathrm{v})$ extraction for $12 \mathrm{~h}$ and oven-dried at $50^{\circ} \mathrm{C}$ for $24 \mathrm{~h}$. The dewaxed sugarcane bagasse was pretreated by a planetary ball-miller for $4 \mathrm{~h}$.

AMIMCl was purchased from Shanghai Cheng Jie Chemical Co. Ltd. (Shanghai, China) and used as received. Methyl acrylate, ethyl acrylate, and butyl acrylate were chemically pure and purchased from Shanghai Ling Feng Chemical Co. Ltd. (Shanghai, China). The acrylates were purified by vacuum distillation prior to use. Potassium persulfate $\left(\mathrm{K}_{2} \mathrm{~S}_{2} \mathrm{O}_{8}\right)$ and ethanol were purchased from Aladdin Industrial Inc. (Shanghai, China). Machine oil, cooking oil, and diesel oil were supplied by Guangzhou Yu Shao Traded Co. Ltd. (Guangzhou, China).

2.2. Preparation of Sugarcane Bagasse Graft Copolymers. Sugarcane bagasse ( $1 \mathrm{~g}$ ) was dispersed in AMIMCl ( $49 \mathrm{~g}$ ) at room temperature. The mixture of sugarcane bagasse/AMIMCl was stirred at $110^{\circ} \mathrm{C}$ under $\mathrm{N}_{2}$ atmosphere for $5 \mathrm{~h}$ to guarantee the complete dissolution. Upon the complete dissolution of sugarcane bagasse, acrylate and potassium persulfate $(0.05 \mathrm{~g}$, $0.185 \mathrm{mmol}$ ) were added into the sugarcane bagasse/AMIMCl 
solution, and the mixture was stirred at $60^{\circ} \mathrm{C}$ for $24 \mathrm{~h}$ to achieve the graft reaction. After the reaction complete, the resulting solution was poured into $500 \mathrm{~mL}$ isopropanol. The precipitant was filtered out, thoroughly washed with isopropanol for five times and then with acetone for three times, and oven-dried at $50^{\circ} \mathrm{C}$ for $24 \mathrm{~h}$ to obtain the sugarcane bagasse graft copolymer. The weight percent gain (WPG) of sugarcane bagasse graft copolymers was calculated according to

$$
\mathrm{WPG}=100 \% \times \frac{\left(M_{1}-M_{0}\right)}{M_{0}},
$$

where $M_{0}$ is the oven dry weight of sugarcane bagasse before dissolving. $M_{1}$ is the oven dry weight of sugarcane bagasse graft copolymers.

2.3. Determination of Oil Absorbency. A weight amount of sugarcane bagasse graft copolymer was immersed in excess oil at room temperature. The sample was taken out from the oil after reaching the swelling equilibrium. The excess oil from the surfaces of the sample was wiped out with filter paper. The oil absorption capacity was calculated by

$$
\text { Oil absorption capacity }=\frac{\left(m-m_{0}\right)}{m_{0}}(\mathrm{~g} / \mathrm{g})
$$

where $m_{0}$ and $m$ are the weights of sample before and after oil absorption, respectively.

2.4. Characterization. Fourier transform infrared spectroscopy (FT-IR) was performed using a BRUKER TENSOR 27 FT-IR spectrophotometer. $\mathrm{KBr}$ disc containing $1 \%$ finely ground samples was applied for the spectrum collection. Thirty-two scans were collected per spectrum at a resolution of $4 \mathrm{~cm}^{-1}$.

For scanning electron microscope (SEM) analysis, the samples were fixed to a metal stub with carbon tapes and coated with gold-palladium using sputter coaters. A LEO 1530VP scanning electron microscope (LEO, Germany) with an accelerating voltage of $10 \mathrm{kV}$ was used to obtain secondary electron images.

To perform the thermogravimetric analysis (TGA), the sample $(9-10 \mathrm{mg})$ was heated from $40^{\circ} \mathrm{C}$ to $600^{\circ} \mathrm{C}$ at a heating rate of $10^{\circ} \mathrm{C} / \mathrm{min}$ in a $\mathrm{Q} 500$ thermogravimetric analyzer (TA Instruments, USA) in nitrogen atmosphere.

\section{Results and Discussion}

3.1. Synthesis of the Sugarcane Bagasse Graft Copolymers. Free radical initiated graft copolymerization was applied to prepare the graft copolymers in the present study. The scheme for acrylate monomers graft copolymerization onto sugarcane bagasse in ionic liquid using $\mathrm{K}_{2} \mathrm{~S}_{2} \mathrm{O}_{8}$ as initiator was shown in Figure 1. As a result of the attachment of the poly(acrylate) side chains onto the sugarcane bagasse fractions, the weight of sugarcane bagasse increases after the graft copolymerization modification. The WPG of control sample (Table 1 , SCB sample) was $-14.5 \%$, suggesting the decomposition of

\begin{tabular}{|c|c|c|c|c|}
\hline \multirow{2}{*}{ Entry } & \multirow{2}{*}{ Monomer } & \multicolumn{2}{|c|}{ Monomer dosage } & \multirow{2}{*}{ WPG/\% } \\
\hline & & Volume/mL & Mole/mmol & \\
\hline SCB & - & 0 & 0 & -14.5 \\
\hline MA1 & Methyl acrylate & 1 & 11.0 & -4.3 \\
\hline MA2 & Methyl acrylate & 2 & 22.1 & -0.7 \\
\hline MA3 & Methyl acrylate & 3 & 33.1 & 9.9 \\
\hline MA4 & Methyl acrylate & 4 & 44.1 & 16.2 \\
\hline EA1 & Ethyl acrylate & 1 & 9.4 & -1.7 \\
\hline EA2 & Ethyl acrylate & 2 & 18.8 & 7.8 \\
\hline EA3 & Ethyl acrylate & 3 & 28.2 & 13.7 \\
\hline EA4 & Ethyl acrylate & 4 & 37.6 & 25.5 \\
\hline BA1 & Butyl acrylate & 1 & 6.9 & 2.4 \\
\hline $\mathrm{BA} 2$ & Butyl acrylate & 2 & 13.9 & 9.1 \\
\hline BA3 & Butyl acrylate & 3 & 20.8 & 10.5 \\
\hline BA4 & Butyl acrylate & 4 & 27.8 & 15.5 \\
\hline
\end{tabular}

TABLE 1: Preparation of sugarcane bagasse graft copolymers.

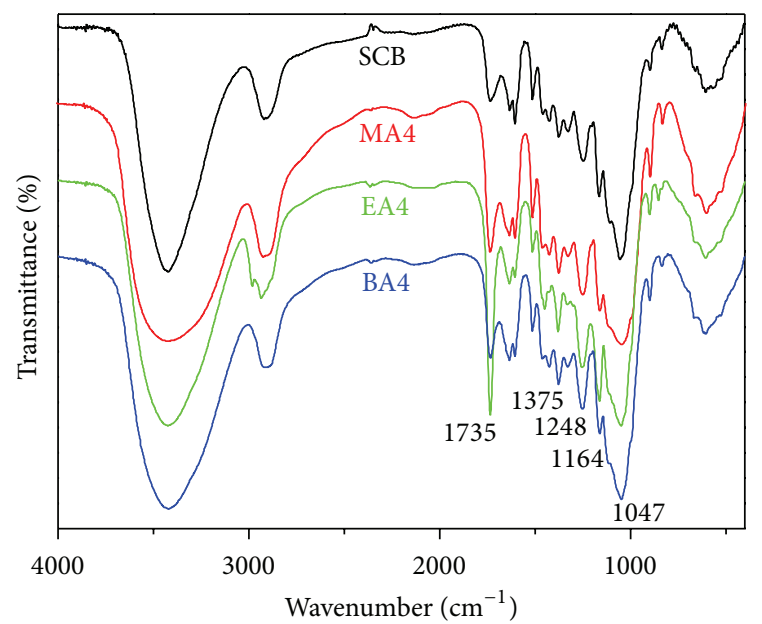

FIGURE 2: FT-IR spectra of sugarcane bagasse and the graft copolymers.

sugarcane bagasse fractions during the dissolution in ionic liquid [15]. The WPG of sugarcane bagasse graft copolymers increased with the increasing monomer dosage, and a series of sugarcane bagasse graft copolymers with WPG in the range of $-5 \%-25.5 \%$ were prepared (Table 1). The WPG of sugarcane bagasse graft poly(ethyl acrylate) was $25.5 \%$ upon the monomer dosage of $4 \mathrm{~mL} / \mathrm{g}$, while it was $16.2 \%$ and $15.5 \%$ for poly(methyl acrylate) and poly(butyl acrylate), respectively. It was suggested that ethyl acrylate was the most reactive monomer for the modification of sugarcane bagasse with free radical initiated graft copolymerization.

3.2. Chemical Structure Confirmed by FT-IR. The FT-IR spectra of native sugarcane bagasse and the graft copolymers are shown in Figure 2. For a better comparability, the spectra were normalized to the band at $1047 \mathrm{~cm}^{-1}$, which is assigned to the glycosidic linkage (C-O-C) stretching vibration in cellulose and hemicelluloses [16]. The characteristic vibrations of the graft poly(acrylates) moieties were identified at $1735 \mathrm{~cm}^{-1}$ 


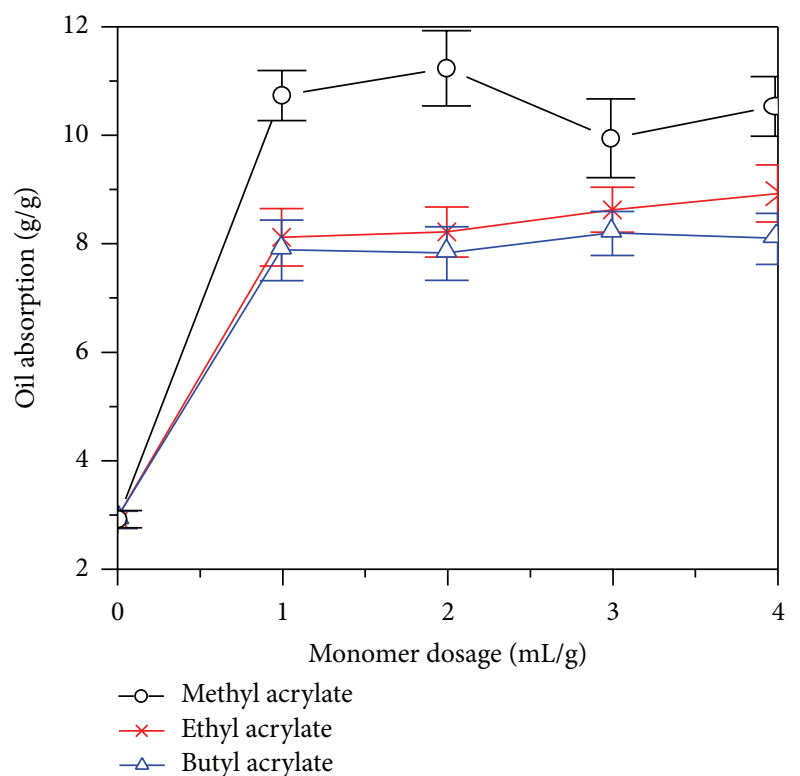

(a)

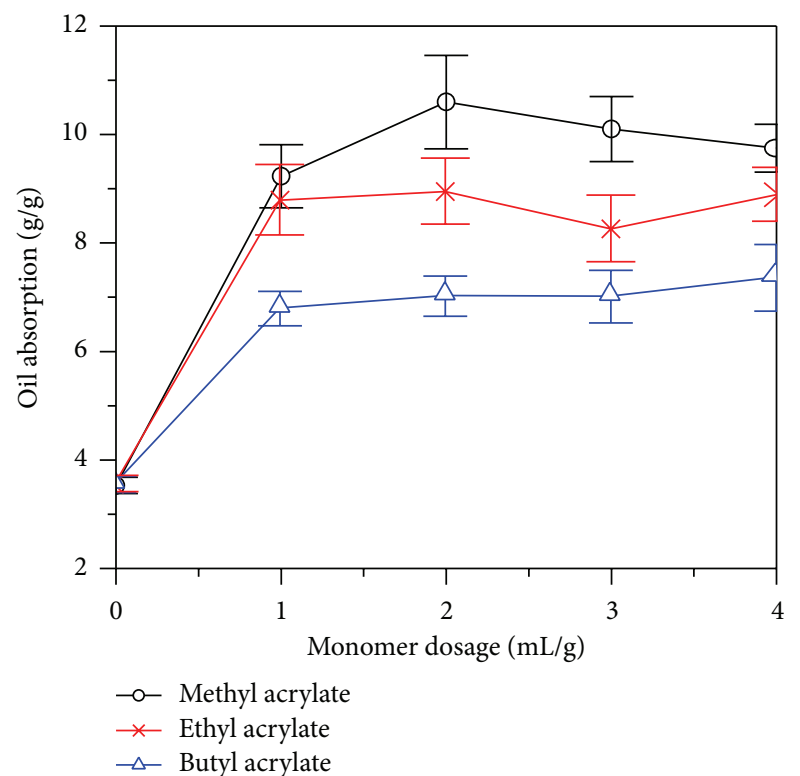

(b)

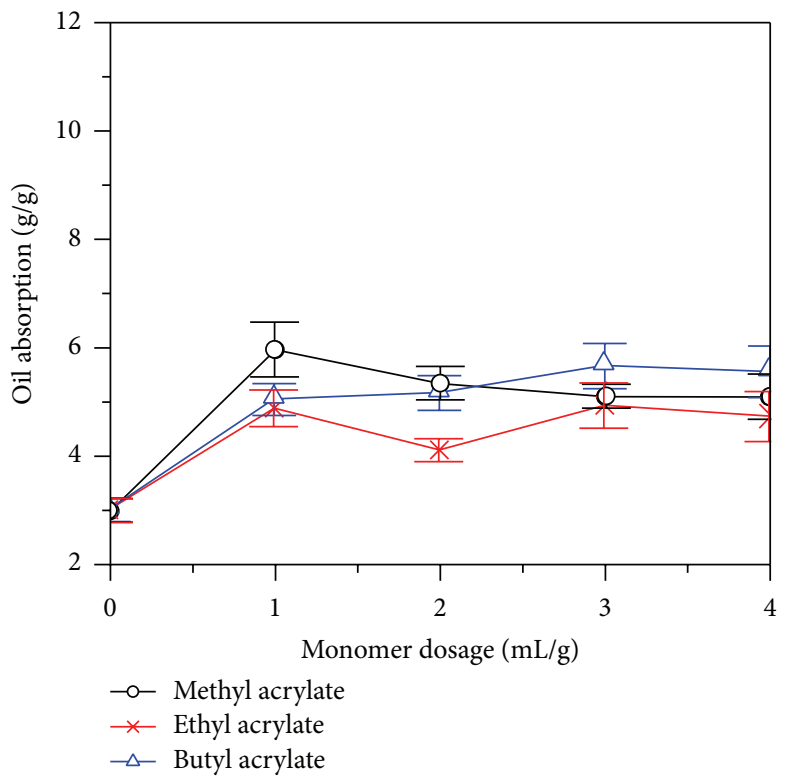

(c)

FIGURE 3: Oil absorption capacity of the sugarcane bagasse graft copolymers. (a) Machine oil absorption capacity; (b) cooking oil absorption capacity; (c) diesel oil absorption capacity.

associated with the carbonyl $(\mathrm{C}=\mathrm{O})$ stretching vibration [17], $1375 \mathrm{~cm}^{-1}$ associated with the methyl $(\mathrm{C}-\mathrm{H})$ deformation vibrations, and $1248 \mathrm{~cm}^{-1}$ and $1164 \mathrm{~cm}^{-1}$ associated with the C-O stretching vibrations $[18,19]$. An increase in the intensity of these bands was observed after the graft copolymerization reaction suggesting the poly(acrylate) side chains were attached onto the sugarcane bagasse successfully.

3.3. Oil Absorption Capacities of the Sugarcane Bagasse Graft Copolymers. The oil absorption capacities of sugarcane bagasse (SCB sample) and the graft copolymers are shown in Figure 3. Clearly, the graft copolymers were more effective at absorbing oil than sugarcane bagasse (SCB sample). Sugarcane bagasse is a natural composite of cellulose, hemicellulose, and lignin with highly crystalline structure and large amount of hydrophilic hydroxyl groups, which make it a poor oil absorption material. After the graft copolymerization with acrylates, the hydrophilic hydroxyl groups of sugarcane bagasse were substituted by the hydrophobic poly(acrylate) side chains. As a result of the substitution of the hydroxyl groups, the modified sugarcane bagasse was more accessible than the unmodified one to oil [20]. 

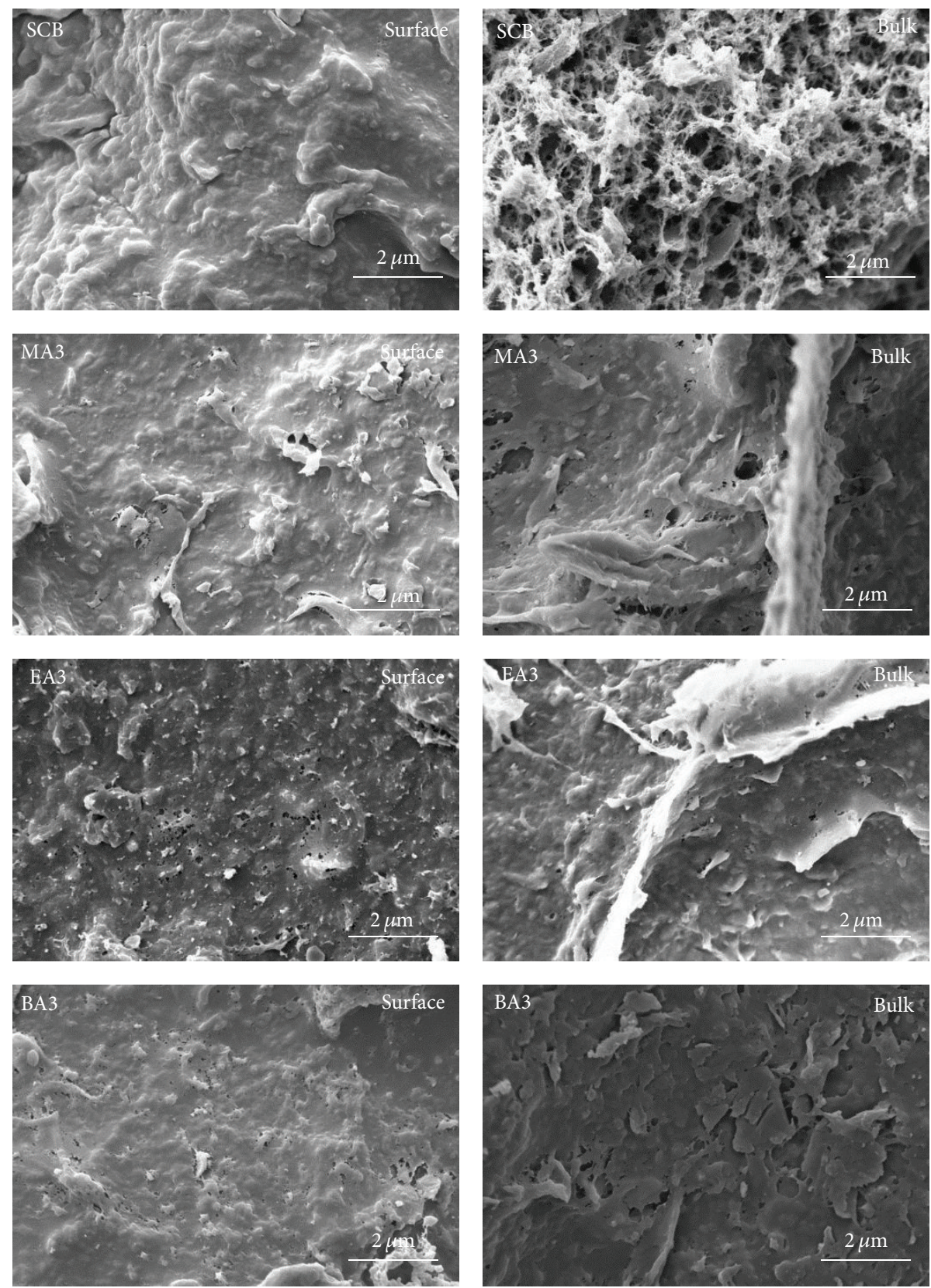

FIGURE 4: SEM photos of the sugarcane bagasse graft copolymers.

It was interesting to find that there was no clear relationship between the oil absorption capacity and the monomer dosage, even though the WPG of graft copolymers increased with the increasing monomer dosage. However, it was reported that the oil absorption capacity increased with the increasing WPG of sugarcane bagasse acetates [21]. In general, the increasing WPG of the sugarcane bagasse acetates corresponds to the increasing degree of substitution (DS) of hydroxyl groups [21], while it is not always the case for the modification of sugarcane bagasse by graft copolymerization. Two chemical reactions, namely, the substitution of hydroxyl groups and the polymerization of the monomers, take place at the same time for the graft copolymerization
[14]. Both increments of DS of hydroxyl groups and degree of polymerization (DP) of side chains would result in the increment of WPG. Increasing the monomer dosage would result in the increment of DP of side chains, while the DS of hydroxyl groups keep unchanged. The WPG of sugarcane bagasse graft copolymers increased with the increasing DP of the poly(acrylate) side chains. However, the oil absorption capacity of the sugarcane bagasse graft copolymers did not change with the increasing monomer dosage, owing to the unchanged DS of hydroxyl groups.

The oil absorption capacities of the graft copolymers differed according to the oil and monomer species. The sugarcane bagasse graft poly(methyl acrylate) showed being 

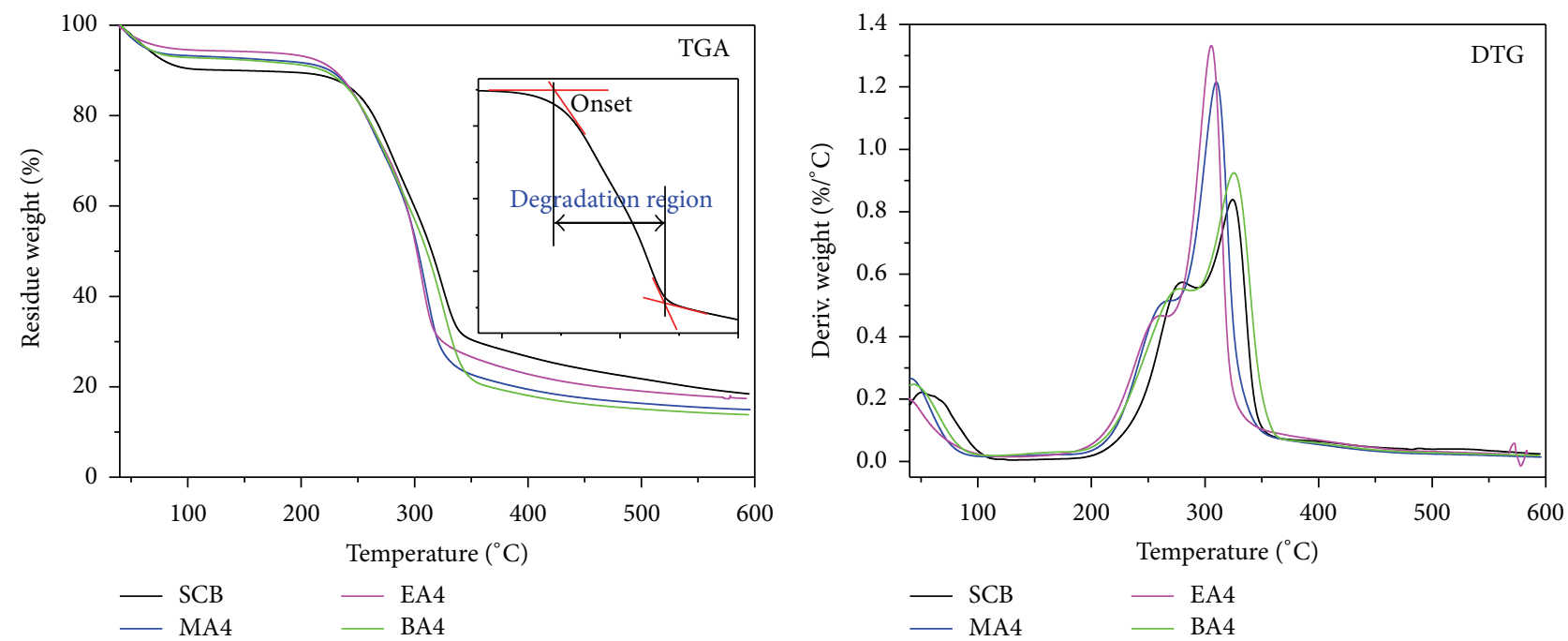

FIGURE 5: TGA/DTG curves of the sugarcane bagasse graft copolymers. The inset figure in TGA showed the method in determining the onset degradation temperature and the degradation region.

the most effective for the absorption of machine oil and cooking oil, while diesel oil absorption capacities of the modified sugarcane bagasse were more or less the same (Figure 3 ). The machine oil and cooking oil were majorly composed of esters, while the diesel was composed of long chain alkane. Acrylates were composed of three parts, namely, allyl group, ester group, and alkane chain ( $\mathrm{R}$ group). The reason why sugarcane bagasse graft poly(acrylate) showed higher absorbency to machine oil and cooking oil than to diesel oil is that both poly(acrylate) and machine/cooking oil contain ester groups. Increasing the chain length of $\mathrm{R}$ group of the acrylates from $\mathrm{CH}_{3}$ to $\mathrm{CH}_{2} \mathrm{CH}_{2} \mathrm{CH}_{2} \mathrm{CH}_{3}$ would result in the decrease of ester group content of sugarcane bagasse graft copolymers. Thus, sugarcane bagasse graft poly(methyl acrylate) had the highest ester content and showed being most efficient for oil absorption.

\subsection{Morphology of the Sugarcane Bagasse Graft Copolymers.} To better understand the enhanced oil absorption behavior of sugarcane bagasse by graft copolymerization modification, the morphology of the sugarcane bagasse derivatives was examined using SEM (Figure 4). The photomicrograph of sugarcane bagasse regenerated from AMIMCl showed a highly porous bulk structure with a homogenous surface (Figure 4, SCB). Both the bulk and surface of sugarcane bagasse graft copolymers showed dense architecture. SEM studies suggested that the oil absorption of sugarcane bagasse was due to the oil penetration into the bulk pores. As a result, the oil absorption of sugarcane bagasse should depend on the pore volume of the material but be independent of oil type. It fitted well with the oil absorbency study that the oil absorption of sugarcane bagasse showed no significant difference for machine oil, cooking oil, and diesel oil. The dense architecture of sugarcane bagasse graft copolymers suggested that the oil absorption of sugarcane bagasse derivatives was not oil held by pores. The introduction of lipophilic groups by graft copolymerization increased oil absorption capacity sugarcane bagasse derivatives.

3.5. Thermal Stability of the Sugarcane Bagasse Graft Copolymers. The TGA/derivative thermogravimetry (DTG) curves of the sugarcane bagasse graft copolymers are shown in Figure 5. The weight loss below $105^{\circ} \mathrm{C}$ was attributed to the volatilization of water. The water content of unmodified sugarcane bagasse (Figure 5, SCB) was $9.7 \%$, and it was $4.7 \%-7.6 \%$ for the modified ones (Figure 5, MA4, EA4, and BA4). It was suggested that the substitution of hydroxyl groups by the hydrophobic poly(acrylate) side chains resulted in the improvement of sugarcane bagasse hydrophobicity. The onset thermal degradation temperature of the unmodified sugarcane bagasse was $244^{\circ} \mathrm{C}$, and it was $228^{\circ} \mathrm{C}$ for all the modified ones. The decrease in the onset thermal degradation temperature was due to the substitution of hydroxyl groups which broke down the hydrogen bonding network of sugarcane bagasse [22]. The degradation region (Figure 5) of the unmodified sugarcane bagasse was $94^{\circ} \mathrm{C}$, and those were $105^{\circ} \mathrm{C}, 96^{\circ} \mathrm{C}$, and $121^{\circ} \mathrm{C}$ for the sugarcane bagasse graft poly(methyl acrylate), poly(ethyl acrylate), and poly(butyl acrylate), respectively. The broadened degradation region of the modified sugarcane bagasse was mainly due to the low onset thermal degradation temperature. The DTG curves suggested that the modified sugarcane bagasse had higher thermal degradation rate than the unmodified one. In summary, the homogeneous graft copolymerization would decrease the thermal stability of modified sugarcane bagasse.

\section{Conclusion}

Lipophilic sugarcane bagasse derivatives were prepared by homogeneous free radical initiated graft copolymerization in ionic liquid. The efficiency of the graft copolymerization was characterized by WPG and controlled by stoichiometric 
methods. The oil absorption capacity of sugarcane bagasse was significantly improved by the graft copolymerization due to the introduction of hydrophobic groups. The oil absorption of sugarcane bagasse graft poly(acrylate) copolymers was attributed to the lipophilic behavior of the samples. The copolymerization processes resulted in the decrease in the thermal stability of sugarcane bagasse by reducing the onset thermal degradation temperature and raising the thermal degradation rate.

\section{Competing Interests}

The authors declare that they have no competing interests.

\section{Acknowledgments}

This work was financially supported by State Key Laboratory of Pulp and Paper Engineering (201602), the Scientific and Technological Project of Guangdong Province (nos. 2013B091500080, 2013B050800023, and 2015A010105019).

\section{References}

[1] L. A. Pfaltzgraff, M. De Bruyn, E. C. Cooper, V. Budarin, and J. H. Clark, "Food waste biomass: a resource for high-value chemicals," Green Chemistry, vol. 15, no. 2, pp. 307-314, 2013.

[2] C. A. Cardona, J. A. Quintero, and I. C. Paz, "Production of bioethanol from sugarcane bagasse: status and perspectives," Bioresource Technology, vol. 101, no. 13, pp. 4754-4766, 2010.

[3] Wikipedia, "Bagasse," 2015, https://en.wikipedia.org/wiki/ Bagasse.

[4] E. Cabane, T. Keplinger, V. Merk, P. Hass, and I. Burgert, "Renewable and functional wood materials by grafting polymerization within cell walls," ChemSusChem, vol. 7, no. 4, pp. 1020-1025, 2014.

[5] Y. C. Fu, G. Li, H. P. Yu, and Y. X. Liu, "Hydrophobic modification of wood via surface-initiated ARGET ATRP of MMA," Applied Surface Science, vol. 258, no. 7, pp. 2529-2533, 2012.

[6] H. P. Yu, Y. C. Fu, G. Li, and Y. X. Liu, "Antimicrobial surfaces of quaternized poly[(2-dimethyl amino)ethyl methacrylate] grafted on wood via ARGET ATRP," Holzforschung, vol. 67, no. 4, pp. 455-461, 2013.

[7] M. A. Ermeydan, E. Cabane, P. Hass, J. Koetz, and I. Burgert, "Fully biodegradable modification of wood for improvement of dimensional stability and water absorption properties by poly( $\varepsilon$-caprolactone) grafting into the cell walls," Green Chemistry, vol. 16, no. 6, pp. 3313-3321, 2014.

[8] I. Kilpeläinen, H. Xie, A. King, M. Granstrom, S. Heikkinen, and D. S. Argyropoulos, "Dissolution of wood in ionic liquids," Journal of Agricultural and Food Chemistry, vol. 55, no. 22, pp. 9142-9148, 2007.

[9] D. A. Fort, R. C. Remsing, R. P. Swatloski, P. Moyna, G. Moyna, and R. D. Rogers, "Can ionic liquids dissolve wood? Processing and analysis of lignocellulosic materials with 1-nbutyl-3-methylimidazolium chloride," Green Chemistry, vol. 9, no. 1, pp. 63-69, 2007.

[10] R. D. Rogers and K. R. Seddon, "Ionic liquids-solvents of the future?” Science, vol. 302, no. 5646, pp. 792-793, 2003.
[11] H. Xie, A. King, I. Kilpelainen, M. Granstrom, and D. S. Argyropoulos, "Thorough chemical modification of wood-based lignocellulosic materials in ionic liquids," Biomacromolecules, vol. 8, no. 12, pp. 3740-3748, 2007.

[12] D. Chen, A.-P. Zhang, C.-F. Liu, and R.-C. Sun, "Modification of sugarcane bagasse with acetic anhydride and butyric anhydride in ionic liquid 1-butyl-3-methylimidazolium chloride," BioResources, vol. 7, no. 3, pp. 3476-3487, 2012.

[13] L. Y. Guo, T. J. Shi, and Z. Li, "Dissolution of fir powder and its graft copolymer in ionic liquid," European Journal of Wood and Wood Products, vol. 69, no. 3, pp. 383-389, 2011.

[14] D. Roy, M. Semsarilar, J. T. Guthrie, and S. Perrier, "Cellulose modification by polymer grafting: a review," Chemical Society Reviews, vol. 38, no. 7, pp. 2046-2064, 2009.

[15] P. Varanasi, P. Singh, M. Auer, P. D. Adams, B. A. Simmons, and S. Singh, "Survey of renewable chemicals produced from lignocellulosic biomass during ionic liquid pretreatment," Biotechnology for Biofuels, vol. 6, no. 1, article 14, 2013.

[16] M. Hauptmann, W. Gindl-Altmutter, C. Hansmann et al., "Wood modification with tricine," Holzforschung, vol. 69, no. 8, pp. 985-991, 2015.

[17] M. Traoré, J. Kaal, and A. Martínez Cortizas, “Application of FTIR spectroscopy to the characterization of archeological wood," Spectrochimica Acta Part A: Molecular and Biomolecular Spectroscopy, vol. 153, pp. 63-70, 2016.

[18] P. Anbinder, C. Macchi, J. Amalvy, and A. Somoza, "Chitosangraft-poly(n-butyl acrylate) copolymer: synthesis and characterization of a natural/synthetic hybrid material," Carbohydrate Polymers, vol. 145, pp. 86-94, 2016.

[19] S. Wang, Q. Wang, X. Fan et al., "Synthesis and characterization of starch-poly(methyl acrylate) graft copolymers using horseradish peroxidase," Carbohydrate Polymers, vol. 136, pp. 1010-1016, 2016.

[20] A. E.-A. A. Said, A. G. Ludwick, and H. A. Aglan, "Usefulness of raw bagasse for oil absorption: a comparison of raw and acylated bagasse and their components," Bioresource Technology, vol. 100, no. 7, pp. 2219-2222, 2009.

[21] X. F. Sun, R. C. Sun, and J. X. Sun, "Acetylation of sugarcane bagasse using NBS as a catalyst under mild reaction conditions for the production of oil sorption-active materials," Bioresource Technology, vol. 95, no. 3, pp. 343-350, 2004.

[22] J. L. Wen, Y. C. Sun, L. Y. Meng, T. Q. Yuan, F. Xu, and R.-C. Sun, "Homogeneous lauroylation of ball-milled bamboo in ionic liquid for bio-based composites production: part I: modification and characterization," Industrial Crops and Products, vol. 34, no. 3, pp. 1491-1501, 2011. 

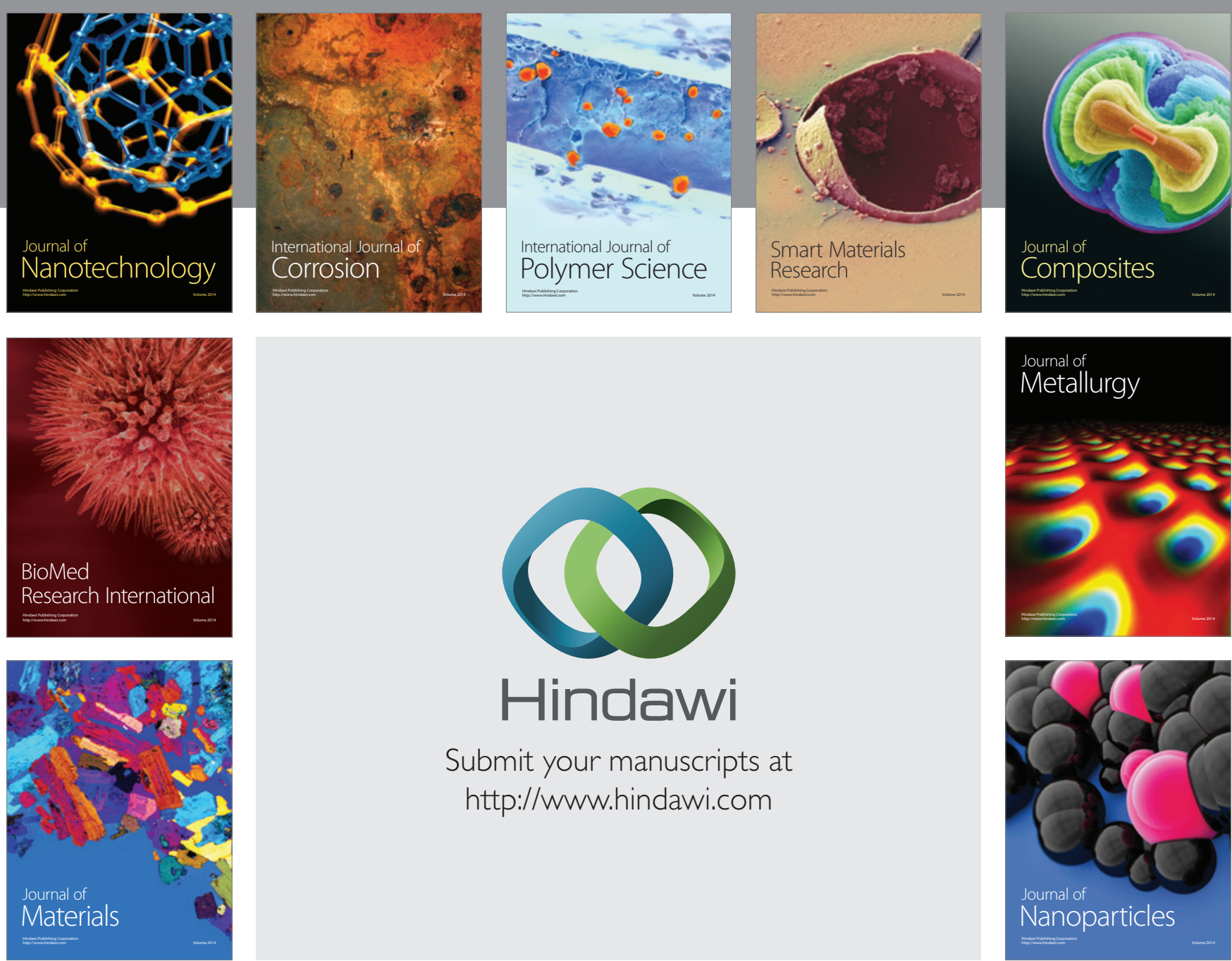

\section{Hindawi}

Submit your manuscripts at

http://www.hindawi.com

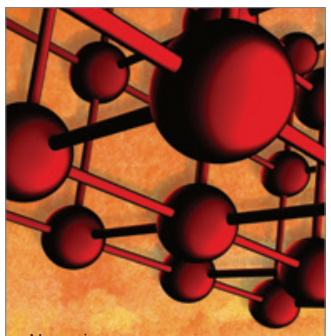

Materials Science and Engineering
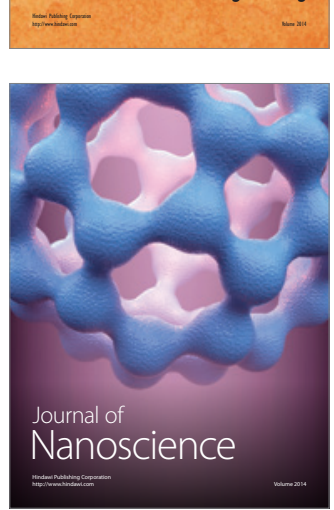
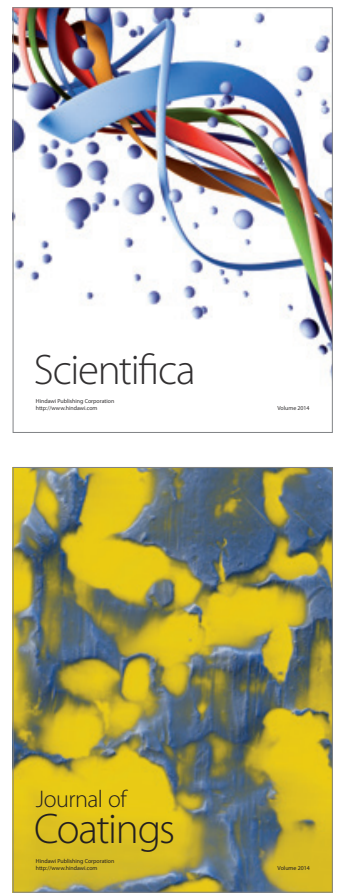
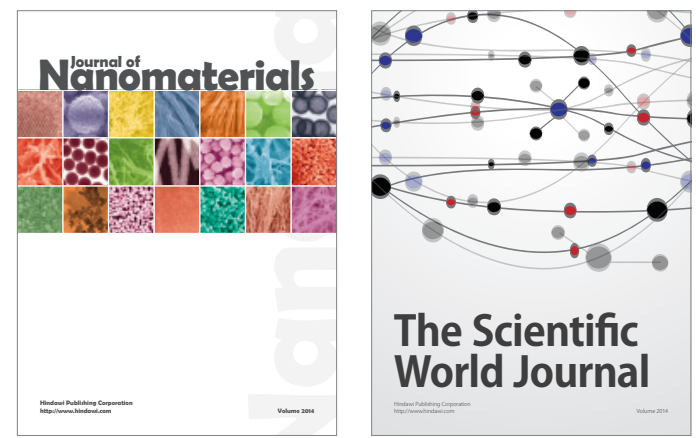

The Scientific World Journal
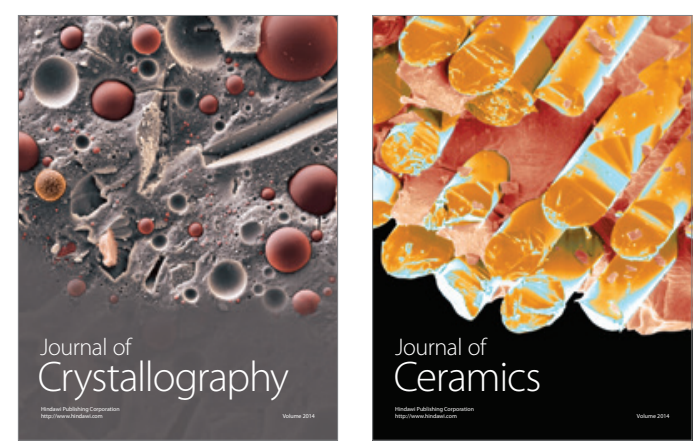
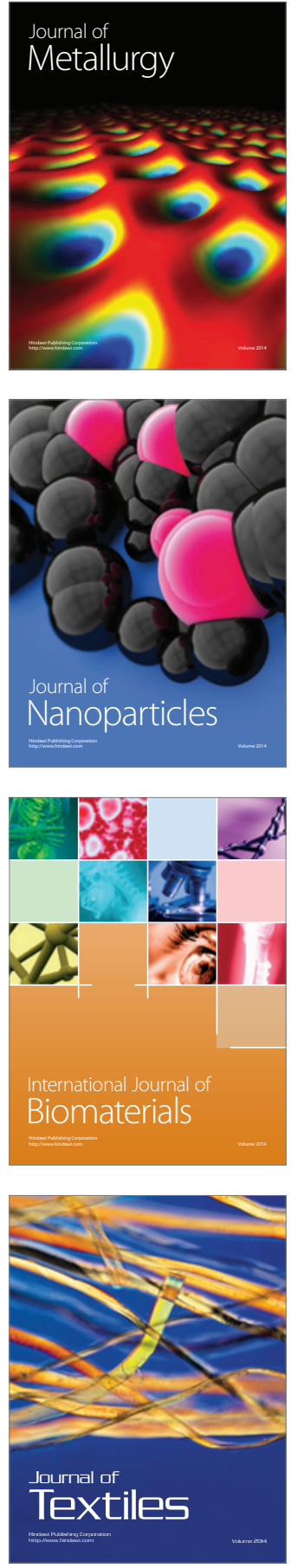